\title{
Impact of Smoking Status on Long-Term Mortality in Patients With Acute Myocardial Infarction
}

\author{
Kunihiro Kinjo, MD; Hiroshi Sato, MD; Yasuhiko Sakata, MD; Daisaku Nakatani, MD; \\ Hiroya Mizuno, MD; Masahiko Shimizu, MD; Tatsuya Sasaki, MD*; \\ Yoshiyuki Kijima, MD**; Masami Nishino, MD ${ }^{\dagger}$; Masaaki Uematsu, MD \\ Jun Tanouchi, MD ${ }^{\dagger}$; Shinsuke Nanto, MD ${ }^{\dagger+}$; Kinya Otsu, MD; Masatsugu Hori, MD \\ on Behalf of the Osaka Acute Coronary Insufficiency Study (OACIS) Group
}

\begin{abstract}
Background Cessation of smoking after a cardiovascular event has been shown in Western countries to have a beneficial effect on clinical events during long-term follow-up. However, knowledge of the effect of smoking status after acute myocardial infarction (AMI) on the long-term mortality based on a large-scale sample is still limited in Japan.

Methods and Results In the present study 2,579 AMI patients were enrolled in the Osaka Acute Coronary Insufficiency Study (OACIS) between April 1998 and March 2003. Smoking status was assessed at baseline and 3 months after hospital discharge by mailed questionnaire. Patients were divided into nonsmokers $(n=823)$, former smokers (those who had stopped smoking before AMI onset, $n=332$ ), quitters (those who stopped smoking after AMI onset, $\mathrm{n}=1,056$ ), and persistent smokers (those who smoked before and after AMI, $\mathrm{n}=368$ ). Quitters had lower long-term mortality rates than persistent smokers (3.0\% vs 5.2\%; log rank, $\mathrm{p}=0.032$ ). Multivariate Cox regression analysis revealed that smoking cessation was independently associated with a reduction in risk of long-term mortality (hazard ratio, 0.39 ; $95 \%$ confidence interval, $0.20-0.77$ ).

Conclusions Patients who continue to smoke after AMI are at greater risk for death than patients who quit smoking. Cessation of smoking benefits the long-term prognosis in patients with AMI. (Circ J 2005; 69: 7-12)
\end{abstract}

Key Words: Mortality; Myocardial infarction; Quitting; Smoking

$\mathbf{S}$ moking is a well-established risk factor for coronary artery disease and acute myocardial infarction (AMI) ${ }^{1-4}$ Cessation of smoking after a cardiovascular event has been shown in Western countries to have a beneficial effect on clinical events during long-term follow-up5-12 Although the Japanese Circulation Society has highlights that nonsmoking measures are extremely important for the prevention and treatment of cardiovascular diseases,$^{13}$ knowledge of the effect of smoking status after AMI on the long-term outcome based on a large-scale sample is still limited in Japan. The purpose of this study was to examine the impact of smoking status on long-term mortality in Japanese AMI patients using a large hospitalbased cohort of patients who survived to hospital discharge.

\section{Methods}

Patients

We registered 4,545 consecutive AMI patients in the

(Received July 30, 2004; revised manuscript received October 27, 2004; accepted October 29, 2004)

Department of Internal Medicine and Therapeutics, Osaka University Graduate School of Medicine, Suita, *Osaka Kosei Nenkin Hospital, Osaka, **Higashi-Osaka City General Hospital, Higashi-Osaka, Osaka Rosai Hospital, Sakai and Hansai Rosai Hospital, Amagasaki, Japan

Mailing address: Hiroshi Sato, MD, PhD, Department of Internal Medicine and Therapeutics, Osaka University Graduate School of Medicine, 2-2 Yamadaoka, Suita 565-0871, Japan. E-mail: satoz@ medone.med.osaka-u.ac.jp
Osaka Acute Coronary Insufficiency Study (OACIS) from April 1998 to March 2003. Of these, 4,035 patients who were still alive at 3 months after hospital discharge were included in the present study. The average duration of follow-up was 885 days. A detailed description of the OACIS has been published elsewhere ${ }^{14-17}$ Briefly, it is a prospective, multicenter observational study in which 25 collaborating hospitals in Japan recorded demographic, procedural, and outcome data and collected blood samples from patients with AMI. The registry was designed to collect uniform, prospective data that could be used to assess clinical variables, therapeutic procedures, and clinical events, ${ }^{14,15}$ and to collect DNA samples that could be used to investigate whether common genetic variations are involved in the pathogenesis of AMI!6,17 The study protocol was approved by the each hospital's ethics committee.

\section{Definitions}

The diagnosis of AMI required the presence of 2 of the following 3 criteria: (1) clinical history of central chest pressure, pain, or tightness lasting for $30 \mathrm{~min}$ or more, (2) typical electrocardiographic changes (ie, ST segment elevation greater than $0.1 \mathrm{mV}$ in at least 1 standard or 2 precordial leads, ST segment depression greater than $0.1 \mathrm{mV}$ in at least 2 leads, abnormal Q wave, or T wave inversion in at least 2 leads), and (3) an increase in the serum creatine kinase activity to more than 2-fold the normal laboratory value. All patients presenting within 1 week of the onset of AMI were registered prospectively, as soon as the diagnosis of AMI was made. 
Table 1 Baseline Characteristics of the Patients With AMI

\begin{tabular}{|c|c|c|c|c|c|}
\hline & $\begin{array}{l}\text { Nonsmokers } \\
\quad(n=823)\end{array}$ & $\begin{array}{l}\text { Former smokers } \\
\qquad(n=332)\end{array}$ & $\begin{array}{c}\text { Quitters } \\
(n=1,056)\end{array}$ & $\begin{array}{c}\text { Persistent smokers } \\
(n=368)\end{array}$ & $p$ value \\
\hline Age, years & 69.2 & $66.0 *$ & $60.9 *,+$ & $60.2 *, \dagger$ & $<0.001$ \\
\hline Male, \% & 52.7 & 94.3 & 89.8 & 89.1 & $<0.001$ \\
\hline Body mass index, $\mathrm{kg} / \mathrm{m}^{2}$ & 23.5 & 23.8 & 23.7 & 23.6 & 0.519 \\
\hline Diabetes mellitus, \% & 34.9 & 35.1 & 29.9 & 32.4 & 0.094 \\
\hline Hypertension, \% & 59.3 & 55.2 & 47.2 & 41.5 & $<0.001$ \\
\hline Hyperlipidemia, \% & 43.1 & 42.0 & 44.8 & 40.2 & 0.471 \\
\hline Prior myocardial infarction, $\%$ & 11.8 & 22.0 & $7.9 *$ & 13.3 & $<0.001$ \\
\hline Prior angina pectoris, $\%$ & 29.4 & 33.1 & 27.1 & 29.9 & 0.114 \\
\hline Prior cerebrovascular disease, $\%$ & 10.0 & 12.6 & 5.3 & 7.4 & $<0.001$ \\
\hline Systolic blood pressure $<100 \mathrm{mmHg}, \%$ & 12.6 & 12.4 & 11.5 & 12.3 & 0.944 \\
\hline Heart rate $\geq 100$ beats $/ \mathrm{min}, \%$ & 13.6 & 11.9 & 8.9 & 7.6 & 0.146 \\
\hline Killip class, $\%$ & & & & & 0.003 \\
\hline 1 & 84.0 & 90.4 & 88.9 & 89.4 & \\
\hline 2 & 10.1 & 6.2 & 6.8 & 6.4 & \\
\hline 3 & 2.8 & 1.9 & 1.5 & 2.5 & \\
\hline 4 & 3.1 & 1.6 & 2.8 & 1.7 & \\
\hline Anterior wall myocardial infarction, \% & 51.0 & 40.4 & 50.6 & 45.7 & 0.003 \\
\hline Atrial fibrillation/flutter, $\%$ & 15.7 & 13.7 & 6.8 & 7.6 & $<0.001$ \\
\hline Ventricular fibrillation/flutter, \% & 9.7 & 8.1 & 13.4 & 10.6 & 0.001 \\
\hline Revascularization therapy, \% & 86.7 & 90.0 & 91.5 & 85.6 & 0.001 \\
\hline Thrombolysis & 8.6 & 13.1 & 13.3 & 9.8 & 0.009 \\
\hline$P C I$ & 82.3 & 84.5 & 86.8 & 81.0 & 0.014 \\
\hline Coronary artery bypass grafting & 2.2 & 1.5 & 1.7 & 1.4 & 0.729 \\
\hline \multicolumn{6}{|l|}{ Medication at discharge, $\%$} \\
\hline ACE inhibitor & 60.0 & 63.5 & 67.2 & 64.9 & 0.015 \\
\hline$\beta$-blocker & 37.7 & 33.1 & 36.5 & 33.4 & 0.345 \\
\hline$H M G-C o A$ reductase inhibitor & 30.3 & 31.3 & 31.1 & 29.6 & 0.943 \\
\hline Antiplatelet agent & 96.8 & 97.3 & 98.2 & 96.5 & 0.174 \\
\hline \multicolumn{6}{|l|}{ Hospital characteristics, $\%$} \\
\hline Performance of $\mathrm{PCI}>200$ procedures/year & 77.3 & 83.8 & 80.1 & 75.5 & 0.007 \\
\hline No. of beds $>200$ & 80.2 & 78.2 & 76.2 & 79.1 & 0.114 \\
\hline AMI case load >50 patients/year & 72.2 & 75.4 & 75.6 & 70.7 & 0.110 \\
\hline
\end{tabular}

ACE, angiotensin-converting enzyme; AMI, acute myocardial infarction; PCI, percutaneous coronary intervention.

$* p<0.001$ vs nonsmokers, ${ }^{t} p<0.001$ vs former smokers.

Table 2 Independent Predictors of Persistent Smoking

\begin{tabular}{lc}
\hline \hline & $\begin{array}{c}\text { Odds ratio } \\
\text { (95\% confidence interval) }\end{array}$ \\
\hline Age & $0.98(0.97-1.00)^{*}$ \\
Male & $0.71(0.44-1.02)$ \\
Body mass index & $1.00(0.95-1.05)$ \\
Diabetes mellitus & $1.11(0.80-1.54)$ \\
Hypertension & $0.75(0.54-1.03)$ \\
Hyperlipidemia & $0.77(0.53-1.11)$ \\
Prior myocardial infarction & $2.04(1.27-3.33)^{\dagger}$ \\
Prior angina pectoris & $1.32(0.94-1.82)$ \\
Prior cerebrovascular disease & $1.67(0.89-3.13)$ \\
Systolic blood pressure $<100$ mmHg & $1.09(0.62-1.67)$ \\
Heart rate $\geq 100$ beats/min & $0.82(0.46-1.47)$ \\
Killip $\geq 2$ & $0.92(0.55-1.54)$ \\
Anterior wall myocardial infarction & $0.93(0.68-1.27)$ \\
Atrial fibrillation/flutter & $0.93(0.52-1.69)$ \\
Ventricular fibrillation/flutter & $0.71(0.43-1.18)$ \\
Revascularization therapy & $0.67(0.42-1.06)$ \\
ACE inhibitor & $1.03(0.74-1.43)$ \\
$\beta$-blocker & $1.02(0.75-1.41)$ \\
HMG-CoA reductase inhibitor & $1.18(0.81-1.72)$ \\
Antiplatelet agent & $0.73(0.29-1.82)$ \\
Performance of PCI $>200$ procedures/year & $0.88(0.42-1.85)$ \\
No. of beds $>200$ & $1.23(0.85-1.79)$ \\
AMI case load $>50$ patients/year & $0.78(0.39-1.56)$ \\
\hline
\end{tabular}

$A C E$, angiotensin-converting enzyme; AMI, acute myocardial infarction; $P C I$, percutaneous coronary intervention. $*_{p}=0.044,{ }^{\dagger} p=0.003$.

\section{Data Collection}

Research cardiologists and specialized research nurses recorded data concerning sociodemographic variables, medical history, therapeutic procedures, and clinical events during the patient's stay in the hospital. Information was obtained from the hospital medical records and by direct interview with the patient, family members, and the treating physician. After each patient gave written informed consent to enter the OACIS, all in-hospital data were transmitted to the data collection center located in the Department of Internal Medicine and Therapeutics in Osaka University Graduate School of Medicine for processing and analysis.

We obtained follow-up clinical data at 3, 6, and 12 months after the onset of AMI and annually thereafter in survivors. We obtained data concerning subsequent cardiac events by visits to the research outpatient clinic or, in a few instances, by verbal or written contact with the patient's physician, the patient, or family members.

\section{Quality Control}

Before initiation of this study, a research cardiologist and specialized research nurse at each site received a training manual that explained how to complete the case report form, defined each variable, and provided examples of correct responses. Double-key entry was used by the data collection center to add each case report form to the database. Audits were performed electronically to detect outof-range variables, inconsistencies, errors, or omissions. 
Table 3 Unadjusted and Adjusted Hazard Ratio of All Cause Death

\begin{tabular}{lcccc}
\hline \hline & $\begin{array}{c}\text { Nonsmokers } \\
(n=823)\end{array}$ & $\begin{array}{c}\text { Former smokers } \\
(n=332)\end{array}$ & $\begin{array}{c}\text { Quitters } \\
(n=1,056)\end{array}$ & $\begin{array}{c}\text { Persistent smokers } \\
(n=368)\end{array}$ \\
\hline Unadjusted $_{\text {Adjusted }}^{*}$ & 1 & $0.90(0.60-1.35)$ & $0.34(0.23-0.50)$ & $0.62(0.38-1.02)$ \\
& 1 & $1.53(0.88-2.66)$ & $0.81(0.45-1.43)$ & $2.27(1.17-4.44)$ \\
\hline
\end{tabular}

*Adjusted for age, gender, body mass index, diabetes mellitus, hypertension, hyperlipidemia, prior myocardial infarction, prior angina pectoris, prior cerebrovascular disease, systolic blood pressure $<100 \mathrm{mmHg}$, heart rate $\geq 100$ beats/min, Killip class $\geq 2$, anterior wall myocardial infarction, atrial fibrillation/flutter, ventricular fibrillation/flutter, revascularization, use of ACE inhibitors, $\beta$-blockers, HMG-CoA reductase inhibitor, and antiplatelet agents, performance of PCI $>200$ procedures/year, no. of beds $>200$, and AMI case load $>50$ patients/year.

$A C E$, angiotensin-converting enzyme; $A M I$, acute myocardial infarction; $P C I$, percutaneous coronary intervention.

Then telephone contact was made with the local research cardiologists and specialized research nurses for resolution of any problems.

\section{Smoking Status}

Smoking status was assessed at baseline on the basis of information obtained from the hospital medical records and by direct interview with the patient, family members, and the treating physician and then at 3 months after hospital discharge on the basis of information provided by a mailed questionnaire. Patients were divided into 4 groups: nonsmokers, defined as patients who had never smoked cigarettes regularly; former smokers, those who had stopped smoking before the onset of AMI; quitters, those who stopped smoking after the onset of AMI; and persistent smokers, those who smoked before and after the onset of AMI.

\section{Statistical Analysis}

The differences in continuous variables between groups were tested by one-way analysis of variance with Bonferroni post hoc test. Categorical variables were compared by the chi-square test. Variables incorporated into our analysis included characteristics previously identified as factors of well-known prognostic importance, including age, gender, body mass index, diabetes mellitus, hypertension, hyperlipidemia, prior myocardial infarction (MI), prior angina pectoris, prior cerebrovascular disease, systolic blood pressure $<100 \mathrm{mmHg}$ on admission, heart rate $\geq 100$ beats/min on admission, Killip class, anterior wall MI, atrial fibrillation/flutter, ventricular fibrillation/flutter, revascularization, medication at discharge (ie, angiotensin-converting enzyme (ACE) inhibitor, $\beta$-blocker, HMG-CoA reductase inhibitor, or antiplatelet agent), and hospital characteristics (ie, no. of percutaneous coronary intervention (PCI) $>200$ procedures/year, no. of beds $>200$, or AMI case load $>50$ patients/year). A multiple logistic regression model was used to analyze factors influencing persistent smoking in patients who were smokers at the time of the incident AMI. Survival curves were constructed by the Kaplan-Meier method and differences in survival were assessed using the $\log$-rank test. Cox regression analysis was used for assessing the relative hazard of events. Analyses of data were performed using statistical software, version 11.0 (SPSS Japan Inc, Tokyo, Japan). For all analyses, significance was defined as $\mathrm{p}<0.05$.

\section{Results}

\section{Baseline Characteristics}

Of the 4,035 AMI patients who were still alive 3 months after hospital discharge, 2,579 completed the 3-month smoking status study. At the time of the incident AMI, 823 patients $(31.9 \%)$ were nonsmokers, 332 patients $(12.9 \%)$ were former smokers, and 1,424 patients $(55.2 \%)$ were current smokers. Of the 1,424 current smokers, 1,056 (74.2\%) had quit smoking and 368 patients $(25.8 \%)$ were persistent smokers at 3 months after hospital discharge. Table 1 shows the baseline characteristics of the study populations. Nonsmokers were older and there was a higher prevalence of women, hypertension, anterior wall MI, atrial fibrillation/flutter, and a higher Killip classes. They were less likely to be treated with reperfusion therapy and ACE inhibitor. The multiple logistic regression model used to analyze factors influencing continuing smoking in patients who were smokers at the time of the incident MI identified younger age and prior MI as independent predictive factors of persistent smokers (Table 2).

\section{Effect of Smoking Status on Long-Term Mortality}

In the unadjusted Cox regression analysis (Table 3), quitters had lower risks of death from all causes than nonsmokers. After adjustment for age, gender, body mass index, diabetes mellitus, hypertension, hyperlipidemia, prior MI, prior angina pectoris, prior cerebrovascular disease, systolic blood pressure $<100 \mathrm{mmHg}$, heart rate $\geq 100$ beats $/ \mathrm{min}$, Killip class $\geq 2$, anterior wall MI, atrial fibrillation/flutter, ventricular fibrillation/flutter, revascularization, use of ACE inhibitors, $\beta$-blockers, HMG-CoA reductase inhibitor, and antiplatelet agents, performance of PCI $>200$ procedures/year, no. of beds $>200$, and AMI case load $>50$ patients/year, persistent smokers had a greater risk of death from all causes than nonsmokers. The hazard ratio for long-term mortality in the quitters was low, equivalent to nonsmokers. To assess the effect of smoking cessation on long-term mortality, we examined the risk of death from all causes in quitters and compared it with the risk in persistent smokers. Fig 1 shows the Kaplan-Meier survival curves for long-term mortality in quitters vs persistent smokers. Quitters had lower long-term all-cause mortality than persistent smokers (3.0\% vs 5.2\%; log rank, $\mathrm{p}=0.032$ ). After adjustment for the previously described variables, smoking cessation was independently associated with a reduction in risk of long-term mortality among patients with AMI (hazard ratio, 0.39; 95\% confidence interval (CI): $0.20-0.77)$.

\section{Discussion}

It is established that cigarette smoking is a major risk factor for coronary artery disease and AMI,-4 and there are several plausible mechanisms for the relation of smoking with worse outcome in patients with AMI. Smoking is associated with increased fibrinogen concentrations, ${ }^{18}$ 


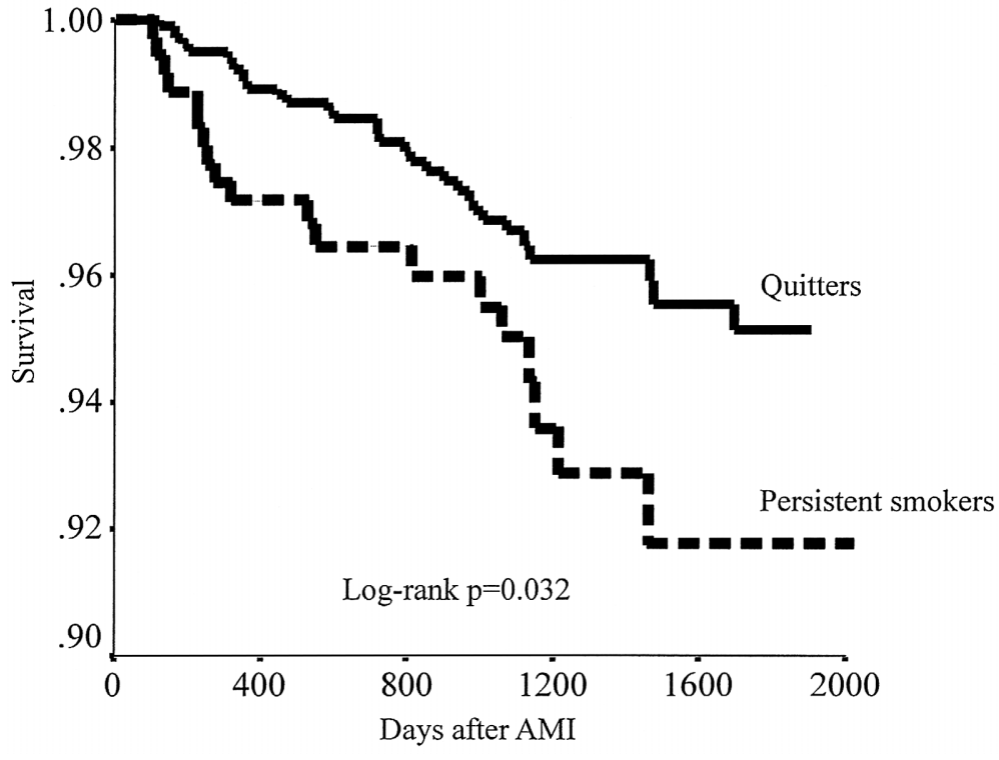

Fig 1. Kaplan-Meier survival curves for long-term mortality according to subsequent smoking status. Quitters had lower long-term all-cause mortality rates than persistent smokers $(3.0 \%$ vs $5.2 \%$; log rank, $\mathrm{p}=0.032$ ). increased platelet aggregability ${ }_{19}^{19}$ reduced fibrinolytic activity, ${ }^{20}$ reduced coronary flow or collateral flow reserve, ${ }^{21,22}$ increased coronary vasoreactivity ${ }^{23}$ and increased myocardial irritability? ${ }^{4}$ However, because the risk of cigarette smoking seems to be reversible, quitting is thought to reduce the recurrent adverse events after AMI ${ }^{25}$ Furthermore, quitters comply more closely with medication schedules and the recommended lifestyle changes than do persistent smokers, ${ }^{26}$ which are also beneficial effects of smoking cessation on long-term mortality after AMI. In fact, the cessation of smoking after AMI has been shown in Western countries to have a beneficial effect on the recurrent adverse events ${ }^{5-11}$ A recent meta-analysis showed that quitting smoking is associated with reduced risk of total mortality and the pooled crude relative risk was 0.64 (95\% CI: 0.58-0.71)!2 In Japan, it has been reported that smoking cessation is associated with reduced second cardiac events (ie, cardiac death and nonfatal MI) in 90 male patients with a history of $\mathrm{MI}^{27}$ but that study had the limitations of small sample size, male subjects only and a combined endpoint. Therefore, we examined the impact of smoking cessation on long-term mortality in Japanese AMI patients using a large hospital-based cohort of patients and found that quitters had lower long-term all-cause mortality rates than persistent smokers and a $61 \%$ reduction in longterm mortality after AMI. Our findings, which are derived from a large-scale sample, suggest that smoking cessation is associated with reduction in mortality in Japanese patients with AMI.

Numerous randomized control trials have established that several therapies reduce the long-term mortality in patients with AMI (ie, ACE inhibitors, $\beta$-blockers, aspirin, and HMG-CoA reductase inhibitors). The reduction in long-term mortality after AMI with the use of ACE inhibitors has been shown to be $23 \% ; 2823 \%$ with $\beta$-blockers; ${ }^{29}$ $15 \%$ with aspirin; ${ }^{30}$ and $29 \%$ with HMG CoA reductase inhibitors ${ }^{31}$ The benefit of smoking cessation $(61 \%$ reduction) appears at least as great as these secondary preventive therapies. Our results suggest that smoking cessation is as effective or more effective for reducing mortality as established secondary preventive therapies. Furthermore, evidence from Western countries had shown that quitting smoking after AMI has considerable economic benefits? ${ }^{2}$
In the present study, $74.2 \%$ of patients had quit smoking at 3 months after hospital discharge. Other studies have reported that the smoking cessation rate is $42-71 \%$ within 6 months after hospital discharge!2,33,34 Although it is difficult to make a simple comparison between present and previous studies, the rate of smoking cessation in our study seems to be equivalent to that in previous studies. However, it has been reported that $70 \%$ of the patients stopped smoking 3 months after AMI, but one-third had resumed smoking after 4 years 34 Therefore, smoking cessation rates in the present study population may decrease after a few years. Smoking cessation interventions have been shown to increase the number of quitters after AMI, but brief interventions are ineffective compared with those initiated during initial hospitalization and accompanied by extended outpatient follow-up 35,36 Therefore, long-term cessation interventions may increase long-term smoking cessation rates and decrease the long-term mortality in our study population.

Although persistent smoking was an independent predictor of long-term mortality, in patients with a prior MI, persistent smokers had higher long-term mortality rates than quitters ( $16.7 \%$ vs $3.7 \%$; log rank, $\mathrm{p}=0.002)$, but in patients with their first MI, long-term mortality did not differ between persistent smokers and quitters $(3.2 \%$ vs $3.1 \%$; log rank, $\mathrm{p}=0.470$ ). Therefore, because a prior MI is an independent predictor of persistent smoking, the recurrence of MI may cause a higher mortality rate in persistent smokers. In fact, a subsequent cardiac event may be fatal because of the severe cardiac dysfunction in patients with a prior MI. Furthermore, patients with a prior MI may be more susceptible to the influence of persistent smoking on mortality than those with a first MI. However, quitting smoking in patients with a prior MI was associated with a reduction of risk in long-term mortality to the same level as patients with a first MI. Therefore, rigorous smoking cessation intervention is required for smokers with a prior MI.

Although randomized control trials are necessary to determine the effect of smoking cessation on long-term mortality after AMI, there are a number of problems associated with the ethics and compliance involved in the random assignment of patients to continue or quitting smoking. In Western countries, smoking cessation counsel- 
ing has been shown to increase smoking cessation rates and to decrease a mortality rates after AMI 35,36 Further studies are needed to determine the effect of counseling on smoking cessation and mortality rates after AMI in Japan.

\section{Study Limitations}

First, because we did not assess the quit date, number of cigarettes smoked per day, and type of smoking, we were not able to determine the relationship of the duration of cessation and smoking reduction to mortality after AMI. Second, there may have been other factors interrelated with smoking behavior that we did not record; for example, social and educational status were not recorded, although differences in either status are very small in Japan. Finally, smoking status was assessed by a self-reported mailed questionnaire and we did not check the smoking behavior by biochemical validation. However, self-reported smoking habits have been found to be accurate in studies of different populations. ${ }^{37}$ Therefore, the influence of misclassification of smoking status on the interpretation of our results was probably limited.

\section{Conclusions}

Persistent smoking is associated with an increased longterm mortality after AMI. However, in patients who quit smoking after AMI, the risk became equivalent to that of nonsmokers. These results support efforts to make smoking cessation after AMI an important goal for both patients and physicians.

\section{Acknowledgments}

This work was supported by a Grant-in-Aid for Scientific Research $(C)$ (2) (\#15590743) from the Japan Society for the Promotion of Science, Tokyo, Japan and by research funds from the Japan Arteriosclerosis Prevention Fund, Tokyo, Japan.

We thank Kumiko Miyoshi, Chizuru Hamaguchi, Hiroko Machida, Mariko Yoneda, Kana Sakatani, Nagisa Yoshioka, Miki Shinkura, Tomomi Miyai, Saeko Kakimoto, Tomoko Inoue, Aki Yabuuchi, and Akiko Yamagishi for their excellent assistance with data collection.

\section{References}

1. Kelly TL, Gilpin E, Ahnve S, Henning H, Ross J Jr. Smoking status at the time of acute myocardial infarction and subsequent prognosis. Am Heart J 1985; 110: 535-541.

2. LaCroix AZ, Lang J, Scherr P, Wallace RB, Cornoni-Huntley J, Berkman L, et al. Smoking and mortality among older men and women in three communities. N Engl J Med 1991; 324: 1619-1625.

3. Miyake Y. Risk factors for non-fatal acute myocardial infarction in middle-aged and older Japanese. Fukuoka heart study group. Jpn Circ J 2000; 64: 103-109.

4. Matsuzaki M, Kita T, Mabuchi H, Matsuzawa Y, Nakaya N, Oikawa $\mathrm{S}$, et al. Large scale cohort study of the relationship between serum cholesterol concentration and coronary events with low-dose simvastatin therapy in Japanese patients with hypercholesterolemia. Circ J 2002; 66: 1087-1095.

5. Wilhelmsson C, Vedin JA, Elmfeldt D, Tibblin G, Wilhelmsen L. Smoking and myocardial infarction. Lancet 1975; 1: 415-420.

6. Mulcahy R, Hickey N, Graham IM, MacAirt J. Factors affecting the 5 year survival rate of men following acute coronary heart disease. Am Heart J 1977; 93: 556-559.

7. Sparrow D, Dawber TR. The influence of cigarette smoking on prognosis after a first myocardial infarction: A report from the Framingham study. J Chronic Dis 1978; 31: 425-432.

8. Salonen JT. Stopping smoking and long-term mortality after acute myocardial infarction. Br Heart J 1980; 43: 463-469.

9. Aberg A, Bergstrand R, Johansson S, Ulvenstam G, Vedin A, Wedel $\mathrm{H}$, et al. Cessation of smoking after myocardial infarction. Effects on mortality after 10 years. Br Heart J 1983; 49: 416-422.

10. Perkins J, Dick TB. Smoking and myocardial infarction: Secondary prevention. Postgrad Med J 1985; 61: 295-300.
11. Rea TD, Heckbert SR, Kaplan RC, Smith NL, Lemaitre RN, Psaty BM. Smoking status and risk for recurrent coronary events after myocardial infarction. Ann Intern Med 2002; 137: 494-500.

12. Critchley JA, Capewell S. Mortality risk reduction associated with smoking cessation in patients with coronary heart disease: A systematic review. JAMA 2003; 290: 86-97.

13. Fujiwara H. Anti-smoking declaration: A message from the Japanese Circulation Society. Circ J 2003; 67: 1-2.

14. Kinjo K, Sato H, Shiotani I, Kurotobi T, Ohnishi Y, Hishida E, et al. Prevalence of helicobacter pylori infection and its link to coronary risk factors in Japanese patients with acute myocardial infarction. Circ J 2002; 66: 805-810.

15. Kinjo K, Sato H, Ohnishi Y, Hishida E, Nakatani D, Mizuno H, et al. Impact of high-sensitivity $\mathrm{C}$-reactive protein on predicting long-term mortality of acute myocardial infarction. Am J Cardiol 2003; 91: 931-935.

16. Ohnishi Y, Tanaka T, Yamada R, Suematsu K, Minami M, Fujii K, et al. Identification of 187 single nucleotide polymorphisms (SNPs) among 41 candidate genes for ischemic heart disease in the Japanese population. Hum Genet 2000; 106: 288-292.

17. Ozaki K, Inoue K, Sato H, Iida A, Ohnishi Y, Sekine A, et al. Functional variation in LGALS2 confers risk of myocardial infarction and regulates lymphotoxin-alpha secretion in vitro. Nature 2004; 429: $72-75$.

18. Wilhelmsen L, Svardsudd K, Korsan-Bengtsen K, Larsson B, Welin $\mathrm{L}$, Tibblin G. Fibrinogen as a risk factor for stroke and myocardial infarction. N Engl J Med 1984; 311: 501-505.

19. Renaud S, Blache D, Dumont E, Thevenon C, Wissendanger T. Platelet function after cigarette smoking in relation to nicotine and carbon monoxide. Clin Pharmacol Ther 1984; 36: 389-395.

20. Newby DE, Wright RA, Labinjoh C, Ludlam CA, Fox KAA, Boon NA, et al. Endothelial dysfunction, impaired endogenous fibrinolysis, and cigarette smoking: A mechanism for arterial thrombosis and myocardial infarction. Circulation 1999; 99: 1411-1415.

21. Deanfield JE, Shea MJ, Wilson RA, Horlock P, de Landsheere CM, Selwyn AP. Direct effects of smoking on the heart: Silent ischemic disturbances of coronary flow. Am J Cardiol 1986; 57: 1005-1009.

22. Czernin J, Sun K, Brunken R, Bottcher M, Phelps M, Schelbert H. Effect of acute and long-term smoking on myocardial blood flow and flow reserve. Circulation 1995; 91: 2891-2897.

23. Sugiishi M, Takatsu F. Cigarette smoking is a major risk factor for coronary spasm. Circulation 1993; 87: 76-79.

24. Mehta MC, Jain AC, Mehta A, Billie M. Cardiac arrhythmias following intravenous nicotine: Experimental study in dogs. J Cardiovasc Pharmacol Ther 1997; 2: 291-298.

25. Gordon T, Kannel WB, McGee D, Dawber TR. Death and coronary attacks in men after giving up cigarette smoking: A report from the Framingham study. Lancet 1974; 2: 1345-1348.

26. Hermanson B, Omenn GS, Kronmal RA, Gersh BJ. Beneficial sixyear outcome of smoking cessation in older men and women with coronary artery disease: Results from the CASS registry. $N$ Engl J Med 1988; 319: 1365-1369.

27. Sato I, Nishida M, Okita K, Nishijima H, Kojima S, Matsumura N, et al. Beneficial effect of stopping smoking on future cardiac events in male smokers with previous myocardial infarction. Jpn Circ J 1992; 56: $217-222$.

28. Flather MD, Yusuf S, Kober L, Pfeffer M, Hall A, Murray G, et al. Long-term ACE-inhibitor therapy in patients with heart failure or left-ventricular dysfunction: A systematic overview of data from individual patients. Lancet 2000; 355: 1575-1581.

29. Freemantle N, Cleland J, Young P, Mason J, Harrison J. Beta blockade after myocardial infarction: Systematic review and meta regression analysis. BMJ 1999; 318: 1730-1737.

30. Collaboration AT. Collaborative meta-analysis of randomised trials of antiplatelet therapy for prevention of death, myocardial infarction, and stroke in high risk patients. BMJ 2002; 324: 71-86.

31. Pignone M, Phillips C, Mulrow C. Use of lipid lowering drugs for primary prevention of coronary heart disease: Meta-analysis of randomised trials. BMJ 2000; 321: $983-986$.

32. Krumholz HM, Cohen BJ, Tsevat J, Pasternak RC, Weinstein MC. Cost-effectiveness of a smoking cessation program after myocardial infarction. J Am Coll Cardiol 1993; 22: 1697-1702.

33. Wilson K, Gibson N, Willan A, Cook D. Effect of smoking cessation on mortality after myocardial infarction: Meta-analysis of cohort studies. Arch Intern Med 2000; 160: 939-944.

34. van Berkel TFM, van der Vlugt MJ, Boersma H. Characteristics of smokers and long-term changes in smoking behavior in consecutive patients with myocardial infarction. Prev Med 2000; 31: 732-741.

35. Dornelas EA, Sampson RA, Gray JF, Waters D, Thompson PD. A randomized controlled trial of smoking cessation counseling after 
myocardial infarction. Prev Med 2000; 30: 261-268.

36. Quist-Paulsen P, Gallefoss F. Randomised controlled trial of smoking cessation intervention after admission for coronary heart disease. BMJ 2003; 327: $1254-1257$.

37. Patrick DL, Cheadle A, Thompson DC, Diehr P, Koepsell T, Kinne S. The validity of self-reported smoking: A review and meta-analysis. Am J Public Health 1994; 84: 1086-1093.

\section{Appendix 1}

The following institutions and persons participated in the OACIS.

Sakurabashi Watanabe Hospital, Osaka, Japan: Fujii K, Ito H; Osaka Police Hospital, Osaka, Japan: Kodama K, Hirayama A; Kansai Rosai Hospital, Amagasaki, Japan: Nagata S, Nanto S, Morozumi T; Ishinkai Yao General Hospital, Yao, Japan: Matsu-ura Y; Osaka General Medical Center, Osaka, Japan: Fukunami M, Shimonagata T; Osaka Rosai Hospital, Sakai, Japan: Yamada Y, Tanouchi J, Nishino M; Kawachi General Hospital, Higashi-Osaka, Japan: Mishima M, Lim YJ; Higashi-Osaka City
General Hospital, Higashi-Osaka, Japan: Kijima Y; Osaka National Hospital, Osaka, Japan: Kusuoka H, Koretsune Y, Yasumura Y; Osaka Minami Medical Center, Kawachinagano, Japan: Kinoshita N, Imai K; Osaka Kosei Nenkin Hospital, Osaka, Japan: Sasaki T; Kobe Ekisaikai Hospital, Kobe, Japan: Shimazu T, Fuji H; Yao Municipal Hospital, Yao, Japan: Hoshida S, Umemoto K; Osaka Railway Hospital of West Japan Railway Company, Osaka, Japan: Ezumi A; Kaizuka City Hospital, Kaizuka, Japan: Morita H, Lee JM; Kita-Osaka Hospital, Osaka, Japan: Ogitani N, Ikeda S; Teramoto Memorial Hospital, Kawachinagano, Japan: Hishida E; Settsu Iseikai Hospital, Settsu, Japan: Akehi N; Kashiwara City Hospital, Kashiwara, Japan: Naka M, Akashi T; Osaka Seamens Insurance Hospital, Osaka, Japan: Kohama A; Saiseikai Senri Hospital, Suita, Japan: Hayashi T, Nakatsuchi Y; Meiwa Hospital, Nishinomiya, Japan: Sugii M; Osaka Kaisei Hospital, Osaka, Japan: Kawabata M; Department of Internal Medicine and Therapeutics, Osaka University Graduate School of Medicine, Suita, Japan: Yamamoto K; Department of Medical Information Science, Osaka University Graduate School of Medicine, Suita, Japan: Takeda H, Matsumura Y. 